\title{
Last Measurable Excretion Rate Normalized by Dose
}

National Cancer Institute

\section{Source}

National Cancer Institute. Last Measurable Excretion Rate Normalized by Dose. NCI

Thesaurus. Code C92392.

The last measurable (positive) excretion rate divided by the dose. 